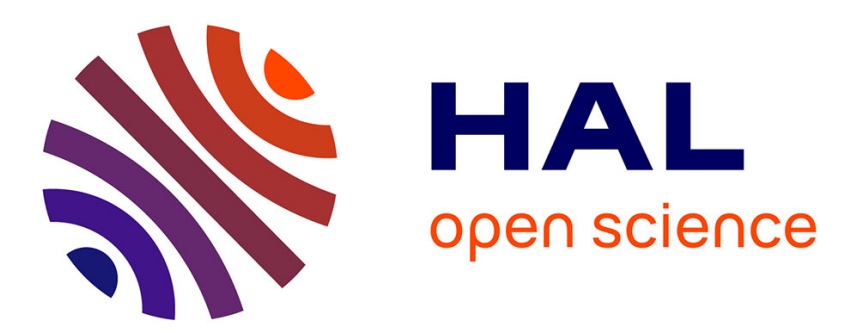

\title{
Differentiability of strongly singular and hypersingular boundary integral formulations with respect to boundary perturbations.
}

Marc Bonnet

\section{- To cite this version:}

Marc Bonnet. Differentiability of strongly singular and hypersingular boundary integral formulations with respect to boundary perturbations.. Computational Mechanics, 1997, 19, pp.240-246. 10.1007/s004660050172 . hal-00111249

\author{
HAL Id: hal-00111249 \\ https://hal.science/hal-00111249
}

Submitted on 9 Aug 2008

HAL is a multi-disciplinary open access archive for the deposit and dissemination of scientific research documents, whether they are published or not. The documents may come from teaching and research institutions in France or abroad, or from public or private research centers.
L'archive ouverte pluridisciplinaire HAL, est destinée au dépôt et à la diffusion de documents scientifiques de niveau recherche, publiés ou non, émanant des établissements d'enseignement et de recherche français ou étrangers, des laboratoires publics ou privés. 


\title{
DIFFERENTIABILITY OF STRONGLY SINGULAR AND HYPERSINGULAR BOUNDARY INTEGRAL FORMULATIONS WITH RESPECT TO BOUNDARY PERTURBATIONS ${ }^{1}$
}

\author{
Marc BonneT \\ Laboratoire de Mécanique des Solides (URA CNRS 317) \\ Ecole Polytechnique, 91128 Palaiseau cedex, France ${ }^{2}$
}

${ }^{1}$ Computational Mechanics, 19, 240-246 (1997).

${ }^{2}$ E-mail address: bonnet@athena.polytechnique.fr 


\begin{abstract}
In this paper, we establish that the Lagrangian-type material differentiation formulas, that allow to express the first-order derivative of a (regular) surface integral with respect to a geometrical domain perturbation, still hold true for the strongly singular and hypersingular syrface integrals usually encountered in boundary integral formulations. As a consequence, this work supports previous investigations where shape sensitivities are computed using the so-called direct differentiation approach in connection with singular boundary integral equation formulations.
\end{abstract}




\section{Introduction}

In e.g. shape design analysis, inverse problems or fracture mechanics, one is often faced with the need of computing sensitivities of integral functional or physical variables with respect to perturbations of the shape of the geometrical domain $\Omega$ under study. This goal is achievable by resorting to either finite-difference methods, considering small but finite domain perturbations, or analytical differentiation followed by discretization.

The analytical approach is a priori clearly superior in terms of both accuracy and efficiency. It relies on either the adjoint variable approach or a direct differentiation of the field equations formulated in weak or BIE fashion. A substantial research effort has been devoted in the last decade or so to various formulations and applications of sensitivity analyses based on analytical differentiation with respect to shape parameters, or on the related mathematical concept of domain derivative. As a result, these concepts are successfully applied to more and more engineering problems. Among a fairly abundant literature, the reader is referred to (Haug et al., 1986), (Simon, 1989), (Sokolowski and Zolesio, 1992), (Dems and Mróz, 1986).

Further, since problems with variable or unknown domains put a great emphasis on the shape of the boundary $\partial \Omega$ of $\Omega$, it is often found convenient, or even essential, to resort to the boundary element method (BEM). Some of these investigations concern the adjoint approach (see e.g. (Burczyński et al., 1995)), which is not discussed further in this paper. Others formulate the direct differentiation approach, which consists of taking the material derivative of the relevant governing boundary integral equation (BIE), so that a governing BIE for the sensitivities of field variables on the boundary is available. The sensitivity of any objective function of interest is then evaluated using (boundary) field variable sensitivities. Both approaches are the main subject of a recent journal special issue, (Bui and Bonnet, 1995).

The material differentiation formula applies a priori to nonsingular or at most weakly singular integrals on moving surfaces. On the other hand, usual BIE formulations are either strongly singular or hypersingular. When regularized BIE formulations are considered, the boundary integrals are at most weakly singular and the material differentiation formula can be applied in a straightforward way. Such formulations are studied, up to second-order material derivatives, in (Bonnet, 1995) for three-dimensional problems and in (Zhang and Mukherjee, 1991) for two-dimensional problems; see also (Matsumoto et al., 1993) and (Nishimura and Kobayashi, 1991). Other authors, e.g. (Barone and Yang, 1989), (Erman and Fenner, 1994), (Mellings and Aliabadi, 1995), apply the material differentiation formula in an equally straightforward way but to strongly singular or hypersingular BIE formulations, without prior regularization. The mathematical validity of this operation, although unquestioned, is not obvious at first. Indeed, the singular BIE formulations are defined as limiting cases of representation formulas when a vanishing neighbourhood of the collocation (singular) point is removed from the domain under consideration. Then, the vanishing neighbourhood is generally affected by the domain perturbations considered for sensitivity analysis, with a priori possible consequences on the result of the limiting process. There lies the source of possible mathematical difficulties when, as in the above references, the effect of domain perturbation on the vanishing neighbourhood is ignored. In the past, failure to take into account a similar effect led to an erroneous value of the free-term associated with the singular representation of strain in the presence of initial strain or stress, until corrected by (Bui, 1978).

The goal of this paper is to put material differentiation of usual singular BIE formulation on safe ground. More precisely, we prove that the usual material differentiation formula for nonsingular surface integrals still yields the correct result when applied to BIE-type strongly singular or hypersingular integrals. In order to do so, the singular integrals are expressed in a fixed (i.e. independent of the domain perturbation) parameter space. The direct approach of (Guiggiani and Gigante, 1990) and (Guiggiani et al., 1992), although initially devised in con- 
nexion with the mapping of a boundary element onto its parent element, is in fact applicable to any regular surface parametrization and provides the key tool for the present analysis. As a result, the usual, straightforward material derivative technique applies without modification to singular BIE formulations. The material derivative of a singular integral takes the form of another singular integral, with the same level of singularity as the initial one. The present analysis encompasses a wide range of singular kernels, including most usual fundamental solutions used in static or dynamic BIE formulations.

\section{Material differentiation}

Let us consider, in the three-dimensional Euclidean space $\mathbb{R}^{3}$ equipped with a Cartesian orthonormal basis $\left(\boldsymbol{e}_{1}, \boldsymbol{e}_{2}, \boldsymbol{e}_{3}\right)$, a body $\Omega_{p}$ whose shape depends on a finite number of shape parameters $\boldsymbol{p}=\left(p_{1}, p_{2}, \ldots\right)$. The latter are treated as time-like parameters using a continuum kinematics-type lagrangian description and introducing an "initial" (nonperturbed) configuration $\Omega=\Omega_{0}$ conventionally associated with $\boldsymbol{p}=\mathbf{0}$ :

$$
\boldsymbol{y} \in \Omega \rightarrow \boldsymbol{y}^{p}=\boldsymbol{\Phi}(\boldsymbol{y} ; \boldsymbol{p}) \in \Omega_{p} \quad \text { where } \quad(\forall \boldsymbol{y} \in \Omega) \mathbf{\Phi}(\boldsymbol{y} ; \mathbf{0})=\boldsymbol{y}
$$

Similarly to continuum kinematics, it is assumed that any such mapping $\boldsymbol{y} \rightarrow \boldsymbol{\Phi}(\boldsymbol{y} ; \boldsymbol{p})$, also termed geometrical transformation, is a diffeomorphism between $\Omega$ and $\Omega_{p}$. A given domain perturbation considered as a whole, as is the case e.g. in shape optimization, admits many different representations (1).

As first-order derivatives with respect to $\boldsymbol{p}$ are considered here, attention is focused without loss of generality to the effect of infinitesimal variations $\delta p$ about $p=0$ of a generic shape parameter $p$ while the others are kept fixed and equal to 0 . The initial transformation velocity $\boldsymbol{\theta}(\boldsymbol{y})$ is defined by

$$
\boldsymbol{\theta}(\boldsymbol{y})=\frac{\partial}{\partial p} \boldsymbol{\Phi}(\boldsymbol{y} ; 0)
$$

In other words, the geometrical transformation (1) has the form

$$
\boldsymbol{y} \rightarrow \boldsymbol{y}^{\delta p}=\boldsymbol{y}+\boldsymbol{\theta}(\boldsymbol{y}) \delta p+\boldsymbol{o}(\delta p)
$$

The material derivative ${ }^{\star}(\boldsymbol{y})$ (also termed total or lagrangian derivative) of a generic field variable $f\left(\boldsymbol{y}^{p}, p\right)$ in the domain transformation, taken at $p=0$, is defined as:

$$
\stackrel{\star}{f}(\boldsymbol{y})=\lim _{\delta p \rightarrow 0} \frac{1}{\delta p}\left[f\left(\boldsymbol{y}^{\delta p}, \delta p\right)-f(\boldsymbol{y}, 0)\right]=\left(\frac{\partial f}{\partial p}+f_{, m} \theta_{m}\right)(\boldsymbol{y}, 0)
$$

using the notation $f_{, m} \equiv \partial f / \partial y_{m}$. Also, the material derivative of a two-point function $K\left(\boldsymbol{y}^{p}-\right.$ $\left.\boldsymbol{x}^{p}\right)$ (e.g. the kernel functions that appear in boundary integral equations), assuming both points $\boldsymbol{x}, \boldsymbol{y}$ follow the geometrical transformation (1), is given by

$$
\stackrel{\star}{K}(\boldsymbol{x}, \boldsymbol{y})=K_{, m}(\boldsymbol{y}-\boldsymbol{x})\left[\theta_{m}(\boldsymbol{y})-\theta_{m}(\boldsymbol{x})\right]
$$

The material derivatives of the unit normal $\boldsymbol{n}$ and the differential area $\mathrm{d} S$ on any moving generic smooth surface $S_{p}$ changing according to (1) are given (Petryk and Mróz, 1986), (Bonnet, 1995) by:

$$
\stackrel{\star}{\mathrm{d}} S=D_{m} \theta_{m} \mathrm{~d} S \quad \stackrel{\star}{\boldsymbol{n}_{i}}=-n_{m} D_{i} \theta_{m}
$$

where

$$
D_{m} f \equiv f_{, m}-n_{m} n_{p} f_{, p}=f_{, m}-n_{m} \frac{\partial f}{\partial n}
$$


denotes the projection of the cartesian partial derivative $\partial f / \partial y_{m}$ onto the tangent plane at $\boldsymbol{y} \in S$. The numbers $D_{i} \theta_{m}$ define in fact the tangential gradient $\boldsymbol{\nabla}_{S} \boldsymbol{\theta}$ while the scalar $D_{m} \theta_{m}$ is the surface divergence of the vector field $\boldsymbol{\theta}$.

The material derivative of a generic regular integral over $S_{p}$ is then given, from e.g. (Petryk and Mróz, 1986), by the formula:

$$
\frac{d}{d p} \int_{S_{p}} f \mathrm{~d} S=\int_{S}\left\{\stackrel{\star}{f}+f D_{m} \theta_{m}\right\} \mathrm{d} S
$$

Note that in formulas (3), (5), (6) and the sequel, all $p$-derivatives are understood for $\boldsymbol{p}=\mathbf{0}$. The argument $p$ is omitted for brevity when $p=0$.

\section{Weakly singular integral on a changing surface}

Let us consider weakly singular integrals of the form

$$
I_{W}(p)=\int_{S_{p}} K^{w}\left(\boldsymbol{y}^{p}-\boldsymbol{x}^{p}\right) u\left(\boldsymbol{y}^{p}, p\right) \mathrm{d} S_{y}
$$

where $u$ is regular and possibly depends on $p$, explicitly and/or implicitly (e.g. $u$ is the field variable which solves a boundary-value problem over the changing domain $\Omega_{p}$ ) and $K^{w}$ is a weakly singular fundamental solution. For three-dimensional potential theory or elastostatic, such kernel functions are usually linear combinations of

$$
K^{w}(\boldsymbol{z})=\frac{z_{i} z_{j}}{|\boldsymbol{z}|^{3}}
$$

where $1 \leq i, j \leq 3$ are fixed indexes; besides, they are homogeneous of degree -1 and symmetric:

$$
K^{w}(a \boldsymbol{z})=\frac{1}{a} K^{w}(\boldsymbol{z}) \quad K^{w}(-\boldsymbol{z})=K^{w}(\boldsymbol{z})
$$

In the BIE context, the surface $S_{p}$ is any portion of the (changing) boundary $\partial \Omega_{p}$ containing the singular point $\boldsymbol{x}^{p}$, which is also assumed to follow the domain transformation (1) (this is emphasized by the chosen notation). It follows that the material derivative of the kernel function $K^{w}$ is given by (4).

Then, application of formula (6) to the weakly singular integral (7) and of (4) leads to:

$$
\begin{aligned}
\frac{d}{d p} I_{W}= & \int_{S} K_{, m}^{w}(\boldsymbol{y}-\boldsymbol{x})\left[\theta_{m}(\boldsymbol{y})-\theta_{m}(\boldsymbol{x})\right] u(\boldsymbol{y}) \mathrm{d} S_{y} \\
& +\int_{S} K^{w}(\boldsymbol{y}-\boldsymbol{x})\left[\stackrel{\star}{u}+u D_{m} \theta_{m}\right](\boldsymbol{y}) \mathrm{d} S_{y}
\end{aligned}
$$

It is important to note that, under the smoothness assumption made on the domain transformation, one has:

$$
|\boldsymbol{\theta}(\boldsymbol{y})-\boldsymbol{\theta}(\boldsymbol{x})| \leq C|\boldsymbol{y}-\boldsymbol{x}|
$$

for some $C>0$. As a consequence, the integrand in eq. (10) is weakly singular, and the straightforward application of the material derivative formula (6) to $I_{W}$ yields the correct result. 


\section{Strongly singular integral on a changing surface}

Let us now consider CPV singular integrals of the form

$$
\begin{aligned}
I_{S}(p) & =\lim _{\varepsilon \rightarrow 0}\left\{\int_{S_{\varepsilon}\left(x^{p}\right)} K^{s}\left(\boldsymbol{y}^{p}-\boldsymbol{x}^{p}\right) \mathrm{d} S_{y}+\int_{S_{p}-e_{\varepsilon}\left(x^{p}\right)} K^{h}\left(\boldsymbol{y}^{p}-\boldsymbol{x}^{p}\right) u\left(\boldsymbol{y}^{p}, p\right) \mathrm{d} S_{y}\right\} \\
& \equiv f_{S_{p}} K^{s}(\boldsymbol{y}-\boldsymbol{x}) u\left(\boldsymbol{y}^{p}, p\right) \mathrm{d} S_{y}
\end{aligned}
$$

and $K^{s}$ is a strongly singular fundamental solution. For three-dimensional potential theory or elastostatics, such kernel functions are usually linear combinations of

$$
K^{s}(\boldsymbol{z})=\frac{z_{i} z_{j} z_{k}}{|\boldsymbol{z}|^{5}}
$$

where $1 \leq i, j, k \leq 3$ are fixed indexes; besides, they are homogeneous of degree -2 and antisymmetric:

$$
K^{s}(a \boldsymbol{z})=\frac{1}{a^{2}} K^{s}(\boldsymbol{z}) \quad K^{s}(-\boldsymbol{z})=-K^{s}(\boldsymbol{z})
$$

When a fundamental solution for flux or traction vector is used in the definition of the integral (12), the unit normal components are included in the nonsingular factor $u$, in order to be still able to invoke the homogeneity property (14a).

In the BIE context, the surface $S_{p}$ is any portion of the (changing) boundary $\partial \Omega_{p}$ containing the singular point $\boldsymbol{x}^{p}$, which is also assumed to follow the domain transformation (1).

CPV integrals are defined as the result of a limiting process where a spherical exclusion neighbourhood $v_{\varepsilon}(\boldsymbol{x})$ of vanishing radius $\varepsilon$ centered at $\boldsymbol{x}$ is removed around $\boldsymbol{x}$. The difficulty that arises when a changing domain is considered is that the geometrical transformation (1) is likely to alter the shape of $v_{\varepsilon}\left(\boldsymbol{x}^{p}\right)$, and this is not allowed by the definition of CPV convergence. Therefore, it is not a priori obvious that a mere application of (6) to (12) yields the correct value for the $p$-derivative of $I_{S}$.

To investigate this issue on a firm ground, we introduce a parametrization of the initial surface $S$ on a parameter space $\Delta$ :

$$
\boldsymbol{\xi}=\left(\xi_{1}, \xi_{2}\right) \in \Delta \rightarrow \boldsymbol{y}(\boldsymbol{\xi}) \in S
$$

This allows the changing surface $S_{p}$ to be mapped onto the fixed parameter space domain $\Delta$ :

$$
(\boldsymbol{\xi} \in \Delta, p \geq 0) \rightarrow \boldsymbol{y}^{p}(\boldsymbol{\xi})=\boldsymbol{\Phi}(\boldsymbol{y}(\boldsymbol{\xi}), p) \in S_{p}
$$

so that one can perform the change of variable $\boldsymbol{y} \rightarrow \boldsymbol{\xi}$ in both $I_{S}(0)$ and $I_{S}(\delta p)$. Of course, $v_{\varepsilon}\left(\boldsymbol{x}^{p}\right)$, or rather $e_{\varepsilon}\left(\boldsymbol{x}^{p}\right)$, is also distorted when mapped onto $\Delta$. However, (Guiggiani and Gigante, 1990) solved this difficulty by giving the correct value of the limiting case $\varepsilon \rightarrow 0$ expressed in the parameter space $\Delta$; their analysis assumes that $\Delta$ is the parent element but in fact remains equally true for any smooth mapping $\Delta \rightarrow S$. As a consequence, a rigorous and computable expression of $I_{S}$ using the parametrization (16) is available for any $p>0$, and indeed provides a sound basis for the present discussion.

The latter result, which we now recall, is based upon the introduction of polar coordinates $(\rho, \alpha)$, centered at the image $\boldsymbol{\eta}$ of the singular point $\boldsymbol{x}^{p}$ in the parameter space $\Delta$

$$
\xi_{1}=\eta_{1}+\rho \cos \alpha \quad \xi_{2}=\eta_{2}+\rho \sin \alpha
$$


so that $\boldsymbol{\xi} \in \Delta \Leftrightarrow 0 \leq \rho \leq \bar{\rho}(\alpha)$ (no loss of generality occurs in assuming that $\Delta$ is star-shaped around $\boldsymbol{\eta}$ ). Since the mapping (16) is smooth, the position vector $\boldsymbol{r}^{p}=\boldsymbol{y}^{p}-\boldsymbol{x}^{p}$ takes the form:

$$
\begin{aligned}
\boldsymbol{r}^{p} & =\rho \hat{\boldsymbol{r}}(\rho, \alpha, p) \\
& =\rho[\boldsymbol{a}(\alpha, p)+o(1)]
\end{aligned}
$$

where

$$
\boldsymbol{a}(\alpha, p)=\hat{\boldsymbol{r}}(0, \alpha, p)=\boldsymbol{a}_{1}(p) \cos \alpha+\boldsymbol{a}_{2}(p) \sin \alpha
$$

and $\left(\boldsymbol{a}_{1}, \boldsymbol{a}_{2}\right)$ is the natural basis, calculated at $\boldsymbol{\xi}=\boldsymbol{\eta}$, associated with the mapping (16):

$$
\boldsymbol{a}_{\beta}(p)=\left.\frac{\partial \boldsymbol{y}^{p}}{\partial \xi_{\beta}}\right|_{\xi=\eta}
$$

An immediate consequence of definition (18) is:

$$
\boldsymbol{a}(\alpha+\pi, p)=-\boldsymbol{a}(\alpha, p)
$$

The kernel function $K^{s}\left(\boldsymbol{r}^{p}\right)$ then takes the form

$$
\begin{aligned}
K^{s}\left(\boldsymbol{r}^{p}\right) & =\frac{1}{\rho^{2}} K^{s}(\hat{\boldsymbol{r}}) \\
& =\frac{1}{\rho^{2}}\left\{K^{s}(\boldsymbol{a})+O(\rho)\right\}
\end{aligned}
$$

The CPV integral (12) is then given, following (Guiggiani and Gigante, 1990), by:

$$
I_{S}(p)=\int_{0}^{2 \pi} \int_{0}^{\bar{\rho}(\alpha)}\left[F(\rho, \alpha, p)-\frac{f(\alpha, p)}{\rho}\right] d \rho d \alpha+\int_{0}^{2 \pi} f(\alpha, p) \ln [\bar{\rho}(\alpha) a(\alpha, p)] d \alpha
$$

using the notations $a(\alpha, p)=|\boldsymbol{a}(\alpha, p)|$ and

$$
\left.\begin{array}{ll}
F(\rho, \alpha, p) & =\frac{1}{\rho} K^{s}(\hat{\boldsymbol{r}}) u\left(\boldsymbol{y}^{p}, p\right) J(\boldsymbol{\xi}, p) \\
f(\alpha, p) & =K^{s}(\boldsymbol{a}) u\left(\boldsymbol{x}^{p}, p\right) J(\boldsymbol{\eta}, p)
\end{array}\right\} \quad\left(\boldsymbol{x}^{p}=\boldsymbol{\Phi}(\boldsymbol{y}(\boldsymbol{\eta}), p), \boldsymbol{y}^{p}=\boldsymbol{\Phi}(\boldsymbol{y}(\boldsymbol{\xi}), p)\right)
$$

$J(\boldsymbol{\xi}, p)$ being the Jacobian of the parametrization (16); $\boldsymbol{a}=\boldsymbol{a}(\alpha, p)$ and $\hat{\boldsymbol{r}}$ are defined by eqs. (17) and (18). Note that the last term in (21) accounts for the preservation of the shape of $v_{\varepsilon}(\boldsymbol{x})$. Besides, the antisymmetry property $(14 \mathrm{~b})$ of $K^{s}$ plays a key role in the validity of the result $(21)\left(I_{S}\right.$ would otherwise be unbounded when $\varepsilon \rightarrow 0$ ) and implies, according to definition (22), that:

$$
f(\alpha+\pi, p)=-f(\alpha, p)
$$

\section{Material derivative of the strongly singular integral}

The formula (21) holds for any fixed $p \geq 0$, the parameter space $\Delta$ being independent of $p$; moreover the double integral is regular because of the singularity removal achieved through the introduction of $f(\alpha, p)$. Thus, the derivative of $I_{S}(p)$ at $p=0$ is obtained using ordinary differentiation of $(21)$ under the integral sign:

$$
\begin{aligned}
\stackrel{\star}{I}_{S}=\frac{d}{d p} I_{S}= & \int_{0}^{2 \pi} \int_{0}^{\bar{\rho}(\alpha)}\left[\stackrel{\star}{F}(\rho, \alpha)-\frac{1}{\rho} \stackrel{\star}{f}(\alpha)\right] d \rho d \alpha+\int_{0}^{2 \pi} \stackrel{\star}{f}(\alpha) \ln [\bar{\rho}(\alpha) a(\alpha)] d \alpha \\
& -\int_{0}^{2 \pi} f(\alpha) \frac{\stackrel{\star}{a}(\alpha)}{a(\alpha)} d \alpha
\end{aligned}
$$


with (using formula (4)):

$$
\begin{aligned}
\stackrel{\star}{F}(\rho, \alpha) & =\frac{1}{\rho} J(\boldsymbol{\xi})\left\{K^{s}(\hat{\boldsymbol{r}})\left[\stackrel{\star}{u}+u D_{m} \theta_{m}\right](\boldsymbol{y})+K_{, m}^{s}(\hat{\boldsymbol{r}})\left[\theta_{m}(\boldsymbol{y})-\theta_{m}(\boldsymbol{x})\right] u(\boldsymbol{y})\right\} \\
\stackrel{\star}{f}(\alpha) & =J(\boldsymbol{\eta})\left\{K^{s}(\boldsymbol{a}) \stackrel{\star}{a}_{i} u(\boldsymbol{x})+\left[\stackrel{\star}{u}+u D_{m} \theta_{m}\right](\boldsymbol{x})\right\}
\end{aligned}
$$

and where all $p$-dependent quantities, like $\hat{\boldsymbol{r}}, \boldsymbol{a}$, are evaluated at $p=0$ (note that $\Delta$, i.e the function $\bar{\rho}(\alpha)$, does not depend on $p$ ). Moreover, the derivatives $\stackrel{\star}{a}$ and $\stackrel{\star}{a}$ are given by

$$
\begin{aligned}
\stackrel{\star}{\boldsymbol{a}}(\alpha) & =\frac{\partial}{\partial p}\left\{\boldsymbol{a}_{1} \cos \alpha+\boldsymbol{a}_{2} \sin \alpha\right\} \\
& =\left.\frac{\partial^{2} \boldsymbol{y}}{\partial \xi_{1} \partial p}\right|_{\xi=\eta} \cos \alpha+\left.\frac{\partial^{2} \boldsymbol{y}}{\partial \xi_{2} \partial p}\right|_{\xi=\eta} \sin \alpha \\
& =\left.\frac{\partial}{\partial \xi_{1}} \frac{\partial \boldsymbol{y}}{\partial p}\right|_{\xi=\eta} \cos \alpha+\left.\frac{\partial}{\partial \xi_{2}} \frac{\partial \boldsymbol{y}}{\partial p}\right|_{\xi=\eta} \sin \alpha \\
& =\left.\frac{\partial \boldsymbol{\theta}}{\partial \xi_{1}}\right|_{\xi=\eta} \cos \alpha+\left.\frac{\partial \boldsymbol{\theta}}{\partial \xi_{2}}\right|_{\xi=\eta} \sin \alpha \\
& =\boldsymbol{\nabla} \boldsymbol{\theta}(\boldsymbol{x}) \cdot \boldsymbol{a}(\alpha) \\
\stackrel{\star}{a}(\alpha) & =\frac{\boldsymbol{a}(\alpha) \cdot \boldsymbol{\nabla} \boldsymbol{\theta}(\boldsymbol{x}) \cdot \boldsymbol{a}(\alpha)}{a(\alpha)}
\end{aligned}
$$

Using the latter formulas and the expansion

$$
\boldsymbol{\theta}(\boldsymbol{y})-\boldsymbol{\theta}(\boldsymbol{x})=\rho \boldsymbol{\nabla} \boldsymbol{\theta}(\boldsymbol{x}) \cdot \boldsymbol{a}+O\left(\rho^{2}\right)=\rho \stackrel{\star}{\boldsymbol{a}}(\alpha)+O\left(\rho^{2}\right)
$$

about $\rho=0$, it is easy to show that ${ }^{\star} / \rho$ is indeed the singular part of $\stackrel{\star}{F}$, and also that $\stackrel{\star}{K}^{s}$ has the same degree of singularity than $K^{s}(\boldsymbol{y}-\boldsymbol{x})$. As a result, $\{\stackrel{\star}{F}-\stackrel{\star}{f} / \rho\}$ is regular. From (25), (26), it is then readily seen that the material derivative $\stackrel{\star}{I}_{S}$ as given by (24) has the form

$$
\stackrel{\star}{I}_{S}=f_{S}\left\{\left[K^{s} u\right]^{\star}+K^{s} u D_{m} \theta_{m}\right\} \mathrm{d} S+R
$$

where the residual term, given by

$$
R=-\int_{0}^{2 \pi} f(\alpha) \frac{\stackrel{\star}{a}(\alpha)}{a(\alpha)} d \alpha
$$

remains to be examinated. Indeed, from (28), one has

$$
f(\alpha) \frac{\stackrel{\star}{a}(\alpha)}{a(\alpha)}=f(\alpha) \frac{\boldsymbol{a}(\alpha) \cdot \boldsymbol{\nabla} \boldsymbol{\theta}(\boldsymbol{x}) \cdot \boldsymbol{a}(\alpha)}{a^{2}(\alpha)}
$$

and then, from property (14b) and the definition (18) of $\boldsymbol{A}(\alpha)$ :

$$
f(\alpha+\pi) \frac{\stackrel{\star}{a}(\alpha+\pi)}{a(\alpha+\pi)}=-f(\alpha) \frac{\stackrel{\star}{a}(\alpha)}{a(\alpha)}
$$

As a result, the residual integral $R(31)$ vanishes:

$$
R=-\int_{0}^{2 \pi} f(\alpha) \frac{\stackrel{\star}{a}(\alpha)}{a(\alpha)} d \alpha=-\left\{\int_{0}^{\pi}+\int_{\pi}^{2 \pi}\right\} f(\alpha) \frac{\stackrel{\star}{a}(\alpha)}{a(\alpha)} d \alpha=0
$$


Thus we have proved that the material differentiation formula (6) is generalizable to CPV singular integrals with the kernel (13):

$$
\begin{aligned}
& \frac{d}{d p}\left\{f_{S_{p}} K^{s}\left(\boldsymbol{y}^{p}-\boldsymbol{x}^{p}\right) u\left(\boldsymbol{y}^{p}, p\right) \mathrm{d} S_{y}\right\} \\
& =f_{S}\left\{\left[K^{s}(\boldsymbol{y}-\boldsymbol{x}) u(\boldsymbol{y})\right]^{\star}+K^{s}(\boldsymbol{y}-\boldsymbol{x}) u(\boldsymbol{y}) D_{m} \theta_{m}(\boldsymbol{y})\right\} \mathrm{d} S_{y}
\end{aligned}
$$

Comments. The result (34) obviously extends for any linear combination of kernels (13); also, the unit normal $\boldsymbol{n}$, if present, can be incorporated without difficulty in the regular factor $u$ and is therefore not excluded from the analysis. All usual potential or isotropic elastostatic strongly singular BIE formulations are encompassed as a direct consequence of the result (34). The analysis presented here assume that $S^{p}$ is smooth at $\boldsymbol{x}^{p}$.

The kernel (13) can be generalized to any combination of kernels of the form $|\boldsymbol{r}|^{-2}\left(\boldsymbol{e}_{r} \otimes \ldots \otimes\right.$ $\boldsymbol{e}_{r}$ ) where the unit vector $\boldsymbol{e}_{r}=\boldsymbol{r} /|\boldsymbol{r}|$ appears an odd number of times in the tensor product, since properties (14) still hold.

The approach of (Barone and Yang, 1989) and others thus receives an a posteriori validation, at least when the free term present in singular BIE formulations remains constant under the geometrical transformation (1). This is normally the case when dealing with initially smooth singular points $\boldsymbol{x}$. Although the present analysis does not answer this issue, it is conjectured that the present result remains true for a corner or edge point $\boldsymbol{x}$, for which the free-term is likely to vary under the geometrical transformation. The fact that the material derivative of a regularized displacement BIE formulation is valid and has the same mathematical form for smooth and corner singular points $\boldsymbol{x}$ ((Bonnet, 1995)) provides in our view an indirect proof of this conjecture.

Finally, the fundamental solutions for dynamic problems (wave propagation, elastodynamics, parabolic heat equation) do not in general verify the homogeneity properties (9a), (14a), (37a). However, their singularity is identical to that of its static counterpart. As a result, upon splitting a dynamic fundamental into the sum of its (singular) static counterpart and a (nonsingular) complement, it is apparent that the result (34) validates the material differentiation of dynamic strongly singular BIE formulations as well.

\section{Hypersingular integral on a changing surface}

Let us now consider the case of hypersingular kernels. Such kernel functions are usually derivatives with respect to source point coordinates of strongly singular kernels:

$$
K_{\ell}^{h}(\boldsymbol{y}-\boldsymbol{x})=\frac{\partial}{\partial x_{\ell}} K^{s}(\boldsymbol{y}-\boldsymbol{x})=-K_{, \ell}^{s}(\boldsymbol{y}-\boldsymbol{x})
$$

In particular, $K_{\ell}^{h}(\boldsymbol{y}-\boldsymbol{x})$ is assumed to be divergence-free:

$$
\frac{\partial}{\partial x_{\ell}} K_{\ell}^{h}(\boldsymbol{y}-\boldsymbol{x})=-K_{\ell, \ell}^{h}(\boldsymbol{y}-\boldsymbol{x})=0 \quad(\boldsymbol{y} \neq \boldsymbol{x})
$$

For three-dimensional potential theory or elastostatics, $K_{\ell}^{h}(\boldsymbol{y}-\boldsymbol{x})$ usually appears as a linear combination of

$$
K^{h}(\boldsymbol{z})=K_{, \ell}^{s}=\frac{4 z_{i} z_{j} z_{k} z_{\ell}}{|\boldsymbol{z}|^{7}}-\frac{\delta i \ell z_{j} z_{k}}{|\boldsymbol{z}|^{5}}-\frac{\delta j \ell z_{i} z_{k}}{|\boldsymbol{z}|^{5}}-\frac{\delta k \ell z_{i} z_{j}}{|\boldsymbol{z}|^{5}}
$$

where $1 \leq i, j, k, \ell \leq 3$ are fixed indexes; besides, they are homogeneous of degree -3 and symmetric:

$$
K_{\ell}^{h}(a \boldsymbol{z})=\frac{1}{a^{3}} K_{\ell}^{h}(\boldsymbol{z}) \quad K_{\ell}^{h}(-\boldsymbol{z})=K_{\ell}^{h}(\boldsymbol{z})
$$


If need be, the unit normal components are included in the nonsingular factor $u$, in order to be still able to invoke the homogeneity property (37a).

Following (Guiggiani et al., 1992), hypersingular boundary integral formulations involve terms defined by a limiting process ('finite part') of the form

$$
\begin{aligned}
I_{H}(p) & =\lim _{\varepsilon \rightarrow 0}\left\{u\left(\boldsymbol{x}^{p}, p\right) \int_{s_{\varepsilon}\left(x^{p}\right)} K^{h}\left(\boldsymbol{y}^{p}-\boldsymbol{x}^{p}\right) \mathrm{d} S_{y}+\int_{S_{p}-e_{\varepsilon}\left(x^{p}\right)} K^{h}\left(\boldsymbol{y}^{p}-\boldsymbol{x}^{p}\right) u\left(\boldsymbol{y}^{p}, p\right) \mathrm{d} S_{y}\right\} \\
& \equiv f_{S} K^{h}(\boldsymbol{y}-\boldsymbol{x}) u(\boldsymbol{y}) \mathrm{d} S_{y}
\end{aligned}
$$

(where again a spherical exclusion neighbourhood $v_{\varepsilon}\left(\boldsymbol{x}^{p}\right)$ of vanishing radius $\varepsilon$ centered at $\boldsymbol{x}^{p}$ is removed around $\left.\boldsymbol{x}^{p}\right)$.

We follow again the line of investigation used in sections 4, 5. From (Guiggiani et al., 1992), the limit (38) is known to exist and a rigorous and computable expression of $I_{H}$ using the parametrization (16) is available for any $p>0$. We now recall the latter result, using again the notations introduced in section 4 , together with some additional notation related to the second-order expansion of the position vector $\boldsymbol{r}$ :

$$
\begin{aligned}
\boldsymbol{r}^{p} & =\rho \hat{\boldsymbol{r}}(\rho, \alpha, p) \\
& =\rho[\boldsymbol{a}(\alpha, p)+\rho \boldsymbol{b}(\alpha, p)+o(\rho)]
\end{aligned}
$$

where

$$
\boldsymbol{b}(\alpha, p)=\boldsymbol{b}_{11}(p) \cos ^{2} \alpha+2 \boldsymbol{b}_{12}(p) \cos \alpha \sin \alpha+\boldsymbol{b}_{22}(p) \sin ^{2} \alpha
$$

and

$$
\boldsymbol{b}_{\alpha \beta}(p)=\left.\frac{\partial^{2} \boldsymbol{y}^{p}}{\partial \xi_{\alpha} \partial \xi_{\beta}}\right|_{\xi=\eta}
$$

Note that formula (40) implies that:

$$
\boldsymbol{b}(\alpha+\pi, p)=\boldsymbol{b}(\alpha, p)
$$

The kernel function $K_{\ell}^{h}\left(\boldsymbol{r}^{p}\right)$ then takes the form

$$
\begin{aligned}
K_{\ell}^{h}\left(\boldsymbol{r}^{p}\right) & =\frac{1}{\rho^{3}} K_{\ell}^{h}(\hat{\boldsymbol{r}}) \\
& =\frac{1}{\rho^{3}}\left\{K_{\ell}^{h}(\boldsymbol{a})+\rho K_{\ell, m}^{h} b_{m}(\alpha, p)+O\left(\rho^{2}\right)\right\}
\end{aligned}
$$

The integral (38) is given by:

$$
\begin{aligned}
I_{H}(p)= & \int_{0}^{2 \pi} \int_{0}^{\bar{\rho}(\alpha)}\left[F_{\ell}(\rho, \alpha, p)-\frac{f_{\ell}(\alpha, p)}{\rho^{2}}-\frac{g_{\ell}(\alpha, p)}{\rho}\right] d \rho d \alpha \\
& +\int_{0}^{2 \pi}\left\{g_{\ell}(\alpha, p) \ln [\bar{\rho}(\alpha) a(\alpha, p)]+f_{\ell}(\alpha, p)\left[\frac{(\boldsymbol{a} . \boldsymbol{b})}{a^{2}}-\frac{1}{\bar{\rho}}\right](\alpha, p)\right\} d \alpha
\end{aligned}
$$

where

$$
\begin{aligned}
F_{\ell}(\rho, \alpha, p) & =\frac{1}{\rho^{2}} K_{\ell}^{h}(\hat{\boldsymbol{r}}) u\left(\boldsymbol{y}^{p}, p\right) J(\boldsymbol{\xi}, p) \\
f_{\ell}(\alpha, p) & =K_{\ell}^{h}(\boldsymbol{a}) u\left(\boldsymbol{x}^{p}, p\right) J(\boldsymbol{\eta}, p) \\
g_{\ell}(\alpha, p) & =K_{\ell, m}^{h}(\boldsymbol{a}) b_{m}(\alpha, p) u\left(\boldsymbol{x}^{p}, p\right) J(\boldsymbol{\eta}, p)+\left.K_{\ell}^{h}(\boldsymbol{a}) \frac{\partial}{\partial \rho}[u J]\right|_{\rho=0}
\end{aligned}
$$


and $\boldsymbol{a}$ and $\hat{\boldsymbol{r}}$ are still defined by eqs. (17) and (18). In fact, $f_{\ell}(\alpha, p)$ and $g_{\ell}(\alpha, p)$ (denoted $F_{-2}$ and $F_{-1}$ in (Guiggiani et al., 1992)) are obtained as the coefficients of $\rho^{0}=1$ and $\rho$ in the Taylor expansion of $K_{\ell}^{h}(\hat{\boldsymbol{r}}) u\left(\boldsymbol{y}^{p}, p\right) J(\boldsymbol{\xi}, p)$ with respect to $\rho$ about $\rho=0$.

The symmetry property (37b) of $K_{\ell}^{h}$, applied to eqs. (45) and (46), imply that:

$$
f_{\ell}(\alpha+\pi, p)=f_{\ell}(\alpha, p) \quad g_{\ell}(\alpha+\pi, p)=-g_{\ell}(\alpha, p)
$$

\section{Material derivative of the hypersingular integral}

The formula (43) holds for any fixed $\boldsymbol{p} \geq \mathbf{0}$, the parameter space $\Delta$ being independent of $\boldsymbol{p}$; moreover the double integral is regular because of the singularity removal achieved through the introduction of $f_{\ell}(\alpha, p)$ and $g_{\ell}(\alpha, p)$. Thus eq. (43) can be differentiated under the integral sign at $p=0$ :

$$
\begin{aligned}
\frac{d}{d p} I_{H}= & \int_{0}^{2 \pi} \int_{0}^{\bar{\rho}(\alpha)}\left[\stackrel{\star}{F}_{\ell}(\rho, \alpha)-\frac{1}{\rho^{2}} \stackrel{\star}{f}{ }_{\ell}(\alpha)-\frac{1}{\rho} \stackrel{\star}{g}_{\ell}(\alpha)\right] d \rho d \alpha \\
& +\int_{0}^{2 \pi}\left\{\stackrel{\star}{g}_{\ell}(\alpha) \ln [\bar{\rho}(\alpha) a(\alpha)]-g_{\ell}(\alpha) \frac{\stackrel{\star}{a}(\alpha)}{a(\alpha)}\right\} d \alpha \\
& +\int_{0}^{2 \pi}\left\{\stackrel{\star}{f}_{\ell}(\alpha)\left[\frac{(\boldsymbol{a} \cdot \boldsymbol{b})}{a^{2}}-\frac{1}{\bar{\rho}}\right](\alpha)+f_{\ell}(\alpha)\left[\frac{(\boldsymbol{a} \cdot \boldsymbol{b})}{a^{2}}-\frac{1}{\bar{\rho}}\right]^{\star}\right\}(\alpha) d \alpha
\end{aligned}
$$

with

$$
\begin{aligned}
\stackrel{\star}{F}(\rho, \alpha)= & \frac{1}{\rho^{2}} J(\boldsymbol{\xi})\left\{K_{\ell, m}^{h}(\hat{\boldsymbol{r}})\left[\theta_{m}(\boldsymbol{y})-\theta_{m}(\boldsymbol{x})\right] u(\boldsymbol{y})+K_{\ell}^{h}(\hat{\boldsymbol{r}})\left[\stackrel{\star}{u}+u D_{m} \theta_{m}\right](\boldsymbol{y})\right\} \\
\stackrel{\star}{f}_{\ell}(\alpha)= & J(\boldsymbol{\eta})\left\{K_{\ell, m}^{h}(\boldsymbol{a}) \stackrel{\star}{a}_{m} u(\boldsymbol{x})+K_{\ell}^{h}(\boldsymbol{a})\left[\stackrel{\star}{u}+u D_{m} \theta_{m}\right](\boldsymbol{x})\right\} \\
\stackrel{\star}{g}_{\ell}(\alpha)= & K_{\ell, m n}^{h}(\boldsymbol{a}) \stackrel{\star}{a}_{n}(\alpha) b_{m}(\alpha) u(\boldsymbol{x}) J(\boldsymbol{\eta}) \\
& +K_{\ell, m}^{h}(\boldsymbol{a})\left(\stackrel{\star}{b}_{m}(\alpha) u(\boldsymbol{x})+b_{m}(\alpha)\left[\stackrel{\star}{u}+u D_{m} \theta_{m}\right](\boldsymbol{x})\right) J(\boldsymbol{\eta}) \\
& +\left.K_{\ell, m}^{h}(\boldsymbol{a}) \stackrel{\star}{a}_{m}(\alpha) \frac{\partial}{\partial \rho}[u J]\right|_{\rho=0}+\left.K_{\ell}^{h}(\boldsymbol{a}) \frac{\partial}{\partial \rho}\left(\left[\stackrel{\star}{u}+u D_{m} \theta_{m}\right] J\right)\right|_{\rho=0}
\end{aligned}
$$

where $\boldsymbol{a}$ and $\hat{\boldsymbol{r}}$ are still defined by eqs. (17) and (18). We note that $\stackrel{\star}{\boldsymbol{a}}$ is given by (27) and that, similarly

$$
\begin{aligned}
\stackrel{\star}{\boldsymbol{b}}(\alpha) & =\frac{\partial}{\partial p}\left\{\boldsymbol{b}_{11} \cos ^{2} \alpha+2 \boldsymbol{b}_{12} \cos \alpha \sin \alpha+\boldsymbol{b}_{22} \sin \alpha\right\} \\
& =\left.\frac{\partial^{2} \boldsymbol{\theta}}{\partial \xi_{1} \partial \xi_{1}}\right|_{\xi=\eta} \cos ^{2} \alpha+\left.2 \frac{\partial^{2} \boldsymbol{\theta}}{\partial \xi_{1} \partial \xi_{2}}\right|_{\xi=\eta} \cos \alpha \sin \alpha+\left.\frac{\partial^{2} \boldsymbol{\theta}}{\partial \xi_{2} \partial \xi_{2}}\right|_{\xi=\eta} \sin ^{2} \alpha
\end{aligned}
$$

Moreover, differentiation of eq. (40) with respect to $p$ gives, accounting for the fact that the variables $p$ and $\xi_{\beta}$ are independent, the following expansion:

$$
\boldsymbol{\theta}(\boldsymbol{y})-\boldsymbol{\theta}(\boldsymbol{x})=\rho[\boldsymbol{\star}(\alpha)+\rho \stackrel{\star}{\boldsymbol{b}}(\alpha)+o(\rho)]
$$

Using the previous formulas, it is then easy to show that

$$
\stackrel{\star}{F}(\rho, \alpha)-\frac{1}{\rho^{2}} \stackrel{\star}{f}_{\ell}(\alpha)-\frac{1}{\rho} \stackrel{\star}{g}_{\ell}(\alpha)=O(1)
$$


is indeed nonsingular. Moreover, using the same argument as in section 5, the antisymmetry property (47b) implies that

$$
\int_{0}^{2 \pi} g_{\ell}(\alpha, p) \frac{\stackrel{\star}{a}(\alpha)}{a(\alpha)} d \alpha=0
$$

Further, since $\bar{\rho}(\alpha)$ does not depend on $p$, one has

$$
\left[\frac{\boldsymbol{a} \cdot \boldsymbol{b}}{a^{2}}-\frac{1}{\bar{\rho}}\right]^{\star}=\left[\frac{\boldsymbol{a} \cdot \boldsymbol{b}}{a^{2}}\right]^{\star}=\frac{\stackrel{\star}{\boldsymbol{a}} \cdot \boldsymbol{b}+\boldsymbol{a} \cdot \stackrel{\star}{\boldsymbol{b}}}{a^{2}}-\frac{2(\boldsymbol{a} \cdot \boldsymbol{b})(\boldsymbol{a} \cdot \stackrel{\star}{\boldsymbol{a}})}{a^{4}}
$$

Then, using eqs. (19), (41), which in turn imply that

$$
\stackrel{\star}{\boldsymbol{a}}(\alpha+\pi)=-\stackrel{\star}{\boldsymbol{a}}(\alpha) \quad \stackrel{\star}{\boldsymbol{b}}(\alpha+\pi)=\stackrel{\star}{\boldsymbol{b}}(\alpha)
$$

it is easy to conclude that

$$
\left[\frac{\boldsymbol{a} . \boldsymbol{b}}{a^{2}}-\frac{1}{\bar{\rho}}\right]^{\star}(\alpha+\pi)=\left[\frac{\boldsymbol{a} \cdot \boldsymbol{b}}{a^{2}}-\frac{1}{\bar{\rho}}\right]^{\star}(\alpha)
$$

and consequently, using (47a), that

$$
\int_{0}^{2 \pi} f_{\ell}(\alpha, p)\left[\frac{(\boldsymbol{a} . \boldsymbol{b})(\alpha, p)}{a^{2}(\alpha, p)}-\frac{1}{\bar{\rho}(\alpha)}\right]^{\star} d \alpha=0
$$

Taking into account all these partial results, eq. (48) reduces to:

$$
\begin{aligned}
\frac{d}{d p} I_{H}= & \int_{0}^{2 \pi} \int_{0}^{\bar{\rho}(\alpha)}\left[\stackrel{\star}{F}_{\ell}(\rho, \alpha)-\frac{1}{\rho^{2}} \stackrel{\star}{f}_{\ell}(\alpha)-\frac{1}{\rho} \stackrel{\star}{g}(\alpha)\right] d \rho d \alpha \\
& +\int_{0}^{2 \pi}\left\{\stackrel{\star}{g}_{\ell}(\alpha) \ln [\bar{\rho}(\alpha) a(\alpha)]+\stackrel{\star}{f}_{\ell}(\alpha)\left[\frac{(\text { a.b })}{a^{2}}-\frac{1}{\bar{\rho}}\right](\alpha)\right\}(\alpha) d \alpha
\end{aligned}
$$

In other words, we have proved that the material differentiation formula (6) is generalizable to hypersingular boundary integrals with kernels (36):

$$
\begin{aligned}
& \frac{d}{d p}\left\{f_{S_{p}} K^{h}\left(\boldsymbol{y}^{p}-\boldsymbol{x}^{p}\right) u\left(\boldsymbol{y}^{p}, p\right) \mathrm{d} S_{y}\right\} \\
& =\neq_{S}\left\{\left[K^{h}(\boldsymbol{y}-\boldsymbol{x}) u(\boldsymbol{y})\right]^{\star}+K^{h}(\boldsymbol{y}-\boldsymbol{x}) u(\boldsymbol{y}) \operatorname{div}_{S} \boldsymbol{\theta}(\boldsymbol{y})\right\} \mathrm{d} S_{y}
\end{aligned}
$$

Comments. The result (54) obviously extends for any linear combination of kernels (36); also, the unit normal $\boldsymbol{n}$, if present, can be incorporated without difficulty in the regular factor $u$ and is therefore not excluded from the analysis. All usual potential or isotropic elastostatic hypersingular BIE formulations are encompassed as a direct consequence of the result (34). All other comments made at the end of section 5 still apply when transposed in the obvious way to the result (34) for hypersingular boundary integrals.

\section{Acknowledgements}

This manuscript has benefited from various discussions with Prof. M. Guiggiani of Siena University, Italy. 


\section{References}

Barone, M. R.; Yang, R. J. 1989: A boundary element approach for recovery of shape sensitivities in threedimensional elastic solids., Comp. Meth. in Appl. Mech. Engng., 74:6982

Bonnet, M. 1995: Regularized BIE formulations for first- and second-order shape sensitivity of elastic fields., Computers and Structures, 56:799-811, (Invited paper, special issue, S. Saigal, guest editor)

Bui, H. D. 1978: Some remarks about the formulation of three-dimensional thermoelastoplastic problems by integral equations., Int. J. Solids Struct., 14:935-939

Bui, H. D.; Bonnet, M., eds. 1995: Unknown or variable domains, inverse problems., vol. 15(2) of Engng. Anal. with Bound. Elem., special issue

Burczyński, T.; Kane, J. H.; Balakrishna, C. 1995: Shape design sensitivity analysis via material derivative - adjoint variable approach., Int. J. Num. Meth. in Eng., 38:2839-2866

Dems, K.; Mróz, Z. 1986: On a class of conservation rules associated with sensitivity analysis in linear elasticity., Int. J. Solids Struct., 22:737-758

Erman, Z.; Fenner, R. T. 1994: Design sensitivity analysis of three-dimensional elastostatic solids using the boundary integral equation method., in C. A. Brebbia, ed., Boundary Element Method XVI., 575-582, Comp. Mech. Publ., Southampton

Guiggiani, M.; Gigante, A. 1990: A general algorithm for multidimensional Cauchy principal value integrals in the boundary element method., ASME J. Appl. Mech., 57:906-915

Guiggiani, M.; Krishnasamy, G.; Rudolphi, T. J.; Rizzo, F. J. 1992: A general algorithm for the numerical solution of hypersingular boundary integral equations., ASME J. Appl. Mech., 59:604-614

Haug, E. J.; Choi, K. K.; Komkov, V. 1986: Design Sensitivity Analysis of Structural Systems, Academic Press

Matsumoto, T.; Tanaka, M.; Miyagawa, M.; Ishii, N. 1993: Optimum design of cooling lines in injection moulds by using boundary element design sensitivity analysis., Finite Elements in Analysis and Design, 14:177-185

Mellings, S. C.; Aliabadi, M. H. 1995: Flaw identification using the boundary element method, Int. J. Num. Meth. in Eng., 38:399-419

Nishimura, N.; Kobayashi, S. 1991: A boundary integral equation method for an inverse problem related to crack detection., Int. J. Num. Meth. in Eng., 32:1371-1387

Petryk, H.; Mróz, Z. 1986: Time derivatives of integrals and functionals defined on varying volume and surface domains., Arch. Mech., 38:694-724

Simon, J. 1989: Second variations for domain optimization problems., in F. Kappel; K. Kunisch; W. Schappacher, eds., Control theory of distributed parameter systems and applications., vol. 91 of International Series of Numerical Mathematics, 361-378, Birkhäuser Verlag, Basel

Sokolowski, J.; Zolesio, J. P. 1992: Introduction to shape optimization. Shape sensitivity analysis, vol. 16 of Springer series in Computational Mathematics, Springer-Verlag 
Zhang, Q.; Mukherjee, S. 1991: Second-order design sensitivity analysis for linear elastic problems by the derivative boundary element method., Comp. Meth. in Appl. Mech. Engng., $86: 321-335$ 